\title{
Real-Time Tracking and Trapping of Single Atoms in Cavity QED
}

\author{
H. J. Kimble, K. Birnbaum, A. C. Doherty, C. J. Hood, T. W.
}

Lynn, H.-C. Nägerl, D. M. Stamper-Kurn, D. W. Vernooy, and J. Ye

Norman Bridge Laboratory of Physics 12-33

California Institute of Technology

Pasadena, California 91125

Cavity quantum electrodynamics (QED) offers powerful possibilities for the deterministic control of atom-photon interactions quantum by quantum [1]. Indeed, modern experiments in cavity QED have achieved the exceptional circumstance of strong coupling, for which single quanta can profoundly impact the dynamics of the atom-cavity system. The diverse accomplishments of this field set the stage for advances into yet broader frontiers in quantum information science for which cavity QED offers unique advantages, such as the realization of quantum networks by way of multiple atom-cavity systems linked by optical interconnects $[2,3]$.

The primary technical challenge on the road toward such scientific goals is the need to trap and localize atoms within a cavity in a setting suitable for strong coupling. Beginning with the work of Mabuchi et al. [4], several groups have been pursuing the integration of the techniques of laser cooling and trapping with those of cavity quantum electrodynamics (QED). [5-12] Two separate experiments in our group have recently achieved significant milestones in this quest, namely the trapping of single atoms in cavity QED $[6,9,10]$. Note that these experiments with cold atoms localized over time $\tau$ achieve $g_{0} \tau \geq 10^{5} \pi$, whereas experiments with conventional atomic beams in cavity QED have $g_{0} T \simeq \pi$, with $T$ as the atomic transit time through the cavity mode.

In our experiments, the arrival of a single atom into the cavity mode can be monitored with high signal-to-noise ratio in real time by a near resonant field with mean intracavity photon number $\bar{n}<1$. We emphasize that interactions in cavity QED bring an in principle enhancement in the capability to sense atomic motion beyond that which is otherwise possible in free space. Stated quantitatively, the ability to sense atomic motion within an optical cavity by way of the transmitted field can be characterized by the optical information $I=\alpha \frac{g_{0}^{2} \Delta t}{\kappa} \equiv \alpha R \Delta t$, which roughly speaking is the maximum possible number of photons that can be collected as signal in time $\Delta t$ with efficiency $\alpha$ as an atom transits between a region of optimal coupling $g_{0}$ and one with $g(\vec{r}) \ll g_{0}$. Here, the coupling parameter $g_{0}$ is defined such that $2 g_{0}$ is the single-photon Rabi frequency and $\kappa$ is the decay rate of the cavity mode itself. A key enabling aspect of our experiments is that $R=\frac{g_{0}^{2}}{\kappa} \gg(\kappa, \gamma)$,

CP551, Atomic Physics 17, edited by E. Arimondo, P. DeNatale, and M. Inguscio (C) 2001 American Institute of Physics 1-56396-982-3/01/\$18.00 
leading to information about atomic motion at a rate that far exceeds that from either cavity decay at rate $\kappa$ or spontaneous scattering at rate $\gamma$ (as in fluorescence imaging).

For our first experiment [13], an atom's arrival within the cavity mode triggers $O N$ an auxiliary field that functions as a far-detuned dipole-force trap (FORT) [9], thereby trapping the atom within the cavity mode. When the FORT is turned $O F F$ after a variable delay, strong coupling likewise enables detection of the atom. Repetition of such measurements for single atoms yields a trap lifetime $\tau=(28 \pm 6) \mathrm{ms}$, which has been limited by fluctuations in the intensity of the FORT arising from the intracavity conversion of FM to AM [14]. Currently implemented improvements in the FM-noise spectrum of the FORT laser should extend this lifetime to well beyond $1 \mathrm{~s}$.

In a second experiment depicted in Figure 1 [15], we rely upon light forces at the single-photon level to trap a single atom within the cavity mode $[6,10]$. As in the preceding experiment, the arrival of a single atom within the resonator is sensed with high signal-to-noise ratio, and triggers a trapping field $O N$. However, in this case, the trapping field is tuned near resonance with the atom-cavity system with $\bar{n} \simeq 1$ intracavity photon. Because the atomic kinetic energy $E_{k}<\hbar g_{0}$, even a single quantum is sufficient to profoundly alter the atomic center-of-mass (CM) motion and indeed to trap an atom as it moves through a region of spatially varying coupling coefficient $g(\vec{r})=g_{0} \psi(\vec{r})$ (as arises in the Gaussian mode of our Fabry-Perot cavity, $\psi(\vec{r}))$.

Furthermore, strong coupling means that the atomic motion will generate large variations in the transmission of a weak probe laser. From the resulting record of the detected photocurrent generated by the transmitted probe, we are able to reconstruct the trajectory of an individual atom by way of a novel inversion algorithm that we have developed. These reconstructions reveal single atoms bound in orbit by the mechanical forces associated with single photons. Our atom-cavity microscope yields $2 \mu \mathrm{m}$ spatial resolution in a $10 \mu \mathrm{s}$ time interval. Over the duration of the observation, the sensitivity is near the standard quantum limit for sensing the motion of a Cesium atom [16].

An important component of this research has been to investigate the extent to which light-induced forces in cavity QED are distinct from their free-space antecedents. The perspective has been to seek qualitatively new manifestations of optical forces at the single-photon level within the setting of cavity QED. Note that quantum character for the relevant fields or phenomena is not ensured the statement that the mean photon number $\bar{n} \sim 1$, since this is trivially the case in an equivalent free-space volume for a field of the same intensity as that inside the cavity.

By way of extensive numerical simulations of the relevant forces and their fluctuations and of comparisons to the standard free-space theory of laser cooling and trapping, we have investigated atom trapping inside optical resonators by the mechanical forces associated with single photons [17]. We have focussed on two points of interest, namely (1) whether or not there are qualitatively different effects of 
optical forces at the single-photon level within the setting of cavity QED, and (2) the features of the resulting atomic motional dynamics and how these dynamics are mapped onto experimentally observable variations of the intracavity field. Not surprisingly, qualitatively distinct atomic dynamics arise as the fundamental coupling and dissipative rates are varied.

For the experiment of Hood et al. [10], our analysis strongly supports the conclusion that atomic motion is largely conservative in nature with only smaller contributions from fluctuating forces. Atomic motion is predominantly in radial orbits transverse to the cavity axis, which in fact enables our reconstruction algorithm. A comparison of the well-known free-space theory and its cavity QED counterpart demonstrates that the usual fluctuations associated with the dipole force along the standing wave are suppressed by an order of magnitude. This suppression in dipole-force heating is based upon the Jaynes-Cummings ladder of eigenstates for the atom-cavity system, which represents qualitatively new physics for optical forces at the single-photon level within the setting of cavity QED [17].

We have also employed our numerical simulations to investigate another experiment that reports atom trapping in the single-photon regime [11]. Somewhat surprisingly, even in a regime of strong coupling, we find that for the parameters of this experiment, there are only small quantitative distinctions between the free-

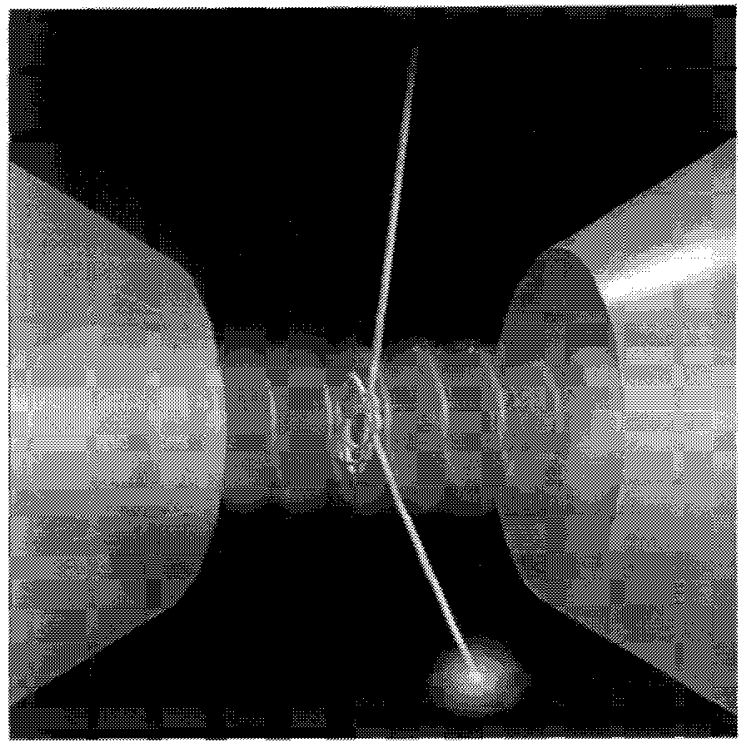

FIGURE 1. Illustration of the tracking and trapping of a single atom with the atom-cavity microscope in a regime with $\vec{n} \simeq 1$ photon. Shown is an experimentally reconstructed trajectory for an atom as it enters into the cavity mode, orbits, and finally exits [10,17]. Animated versions of reconstructed atom orbits can be viewed at www.its.caltech.edu/ qoptics/atomorbits/. 
space theory and the appropriate quantum theory in cavity QED. It is thus not at all clear that description of this experiment as a novel single-quantum trapping effect is necessary [17]. Furthermore, in this setting our simulations demonstrate that atomic motion is dominated by diffusion-driven fluctuations in both the radial and axial dimensions, leading to an average observed localization time comparable to the time for an atom to transit freely through the cavity. The non-conservative character of the dynamics also hampers and often prohibits inference of atomic motion from the record of intracavity photon number, for both radial and axial processes.

To conclude, we emphasize again our goal of utilizing interactions in cavity QED to enable diverse protocols in quantum information science $[2,3]$. Our initial realizations of trapped atoms in cavity QED are important steps towards these ends. This research has been supported by the NSF, by DARPA via the QUIC (Quantum Information and Computation) program administered by ARO, and by the ONR.

\section{REFERENCES}

1. For a recent review, see contributions in the Special Issue of Physica Scripta T76 (1998).

2. J.-I. Cirac, et al., Physica Scripta T76, 223 (1998).

3. A. S. Parkins and H. J. Kimble, Journal Opt. B: Quantum Semiclass. Opt. 1, 496 (1999).

4. H. Mabuchi, Q. A. Turchette, M. S. Chapman, and H. J. Kimble, Opt. Lett. 21, $1393(1996)$.

5. C. J. Hood, M. S. Chapman, T. W. Lynn, and H. J. Kimble, Phys. Rev. Lett. 80, 4157 (1998).

6. J. Ye, C. J. Hood, T. Lynn, H. Mabuchi, D. W. Vernooy, and H. J. Kimble, IEEE Trans. Instru. \& Meas. 48, 608 (1999).

7. H. Mabuchi, J. Ye, and H. J. Kimble, Appl. Phys. B 68, 1095 (1999).

8. P. Münstermann, T. Fischer, P. Maunz, P. W. H. Pinkse, and G. Rempe, Phys. Rev. Lett. 82, 3791 (1999).

9. J. Ye, D. W. Vernooy, and H. J. Kimble, Phys. Rev. Lett. 83, 4987 (1999).

10. C. J. Hood, T. W. Lynn, A. C. Doherty, A.S. Parkins, and H. J. Kimble, Science 287, 1447 (2000).

11. P. W. H. Pinkse, T. Fischer, P. Maunz, and G. Rempe, Nature 404, 365 (2000).

12. P. Münstermann, T. Fischer, P. Maunz, P. W. H. Pinkse, and G. Rempe, Phys. Rev. Lett. 84, 4068 (2000).

13. In this experiment, the Fabry-Perot cavity into which the atoms fall is formed from two super-polished spherical mirrors. The cavity length $l=44.6 \mu \mathrm{m}$, waist $w_{0}=$ $20 \mu \mathrm{m}$, and finesse $F=4.2 \times 10^{5}$, and hence a cavity field decay rate $\kappa / 2 \pi=4 \mathrm{MHz}$. The atomic transition employed for cavity QED is the $\left(g \equiv 6 S_{1 / 2}, F=4, m_{F}=\right.$ $4 \rightarrow e \equiv 6 P_{3 / 2}, F=5, m_{F}=5$ ) component of the $D_{2}$ line of atomic Cesium at $\lambda_{\text {atom }} \equiv c / \nu_{\text {atom }}=852.4 \mathrm{~nm}$. For our cavity geometry and from the atomic transition properties, we have $\left(g_{0}, \gamma_{\perp}\right) / 2 \pi=(32,2.6) \mathrm{MHz}$, with $g_{0}$ as the peak atom-field 
coupling coefficient and $\gamma_{\perp}$ as the dipole decay rate for the $e \rightarrow g$ transition. These rates lead to critical photon and atom numbers $\left(m_{0} \equiv \gamma_{\perp}^{2} / 2 g_{0}^{2}, N_{0} \equiv 2 \kappa \gamma_{\perp} / g_{0}^{2}\right)=$ $(0.003,0.02)$.

14. C. W. Gardiner, J. Ye, H.-C. Nägerl and H. J. Kimble, Phys. Rev. A6104, 5801 (2000).

15. The optical cavity for this experiment is formed by two $1 \mathrm{~mm}$ diameter, $10 \mathrm{~cm}$ radius of curvature mirrors, located on the tapered end of $4 \mathrm{~mm} \mathrm{x} 3 \mathrm{~mm}$ glass substrates. The multi-layer dielectric mirror coatings have a transmission of $4.5 \times 10^{-6}$ and absorption/scatter losses of $2.0 \times 10^{-6}$, giving rise to a cavity finesse $F=480,000$. For the measured cavity length $l=10.9 \mu \mathrm{m}$ and waist $w_{0}=14 \mu \mathrm{m}$, we have parameters $\left(g_{0}, \kappa, \gamma\right)=2 \pi(110,14.2,2.6) \mathrm{MHz}$, where again these rates refer to the transition $\left\{6 S_{1 / 2}, F=4, m_{F}=4\right\} \rightarrow\left\{6 P_{3 / 2}, F=5, m_{F}=5\right\}$ at $\lambda=852 \mathrm{~nm}$ in atomic Cesium. These rates lead to critical photon and atom numbers $\left(m_{0} \equiv \gamma_{\perp}^{2} / 2 g_{0}^{2}, N_{0} \equiv\right.$ $\left.2 \kappa \gamma_{\perp} / g_{0}^{2}\right)=(0.00028,0.0061)$.

16. A more extensive gallery of reconstructed orbits can be viewed at http://www.its.caltech.edu/ qoptics/atomorbits/gallery.html.

17. A. C. Doherty, T. W. Lynn, C. J. Hood, and H. J. Kimble, submitted to Phys. Rev. A, available as quant-ph/0006015. 